This is the author's final, peer-reviewed manuscript as accepted for publication. The publisher-formatted version may be available through the publisher's web site or your institution's library.

\title{
Wave scattering by many small bodies: transmission boundary conditions
}
A. G. Ramm

\section{How to cite this manuscript}

If you make reference to this version of the manuscript, use the following information:

Ramm, A. G. (2013). Wave scattering by many small bodies: Transmission boundary conditions. Retrieved from http://krex.ksu.edu

\section{Published Version Information}

Citation: Ramm, A. G. (2013). Wave scattering by many small bodies: Transmission boundary conditions. Reports on Mathematical Physics, 71(3), 279-290.

Copyright: Copyright @ 2013 Polish Scientific Publishers. Published by Elsevier Ltd.

Digital Object Identifier (DOI): doi:10.1016/S0034-4877(13)60033-6

Publisher's Link: http://www.sciencedirect.com/science/article/pii/S0034487713600336

This item was retrieved from the K-State Research Exchange (K-REx), the institutional repository of Kansas State University. K-REx is available at http://krex.ksu.edu 
Reports on Mathematical Physics, Vol.71, 2013, no. 3, 279- - 290

\title{
Wave scattering by many small bodies: transmission boundary conditions
}

\author{
A. G. Ramm \\ †Mathematics Department, Kansas State University, \\ Manhattan, KS 66506-2602, USA \\ email: ramm@math.ksu.edu
}

\begin{abstract}
Wave scattering by many $(M=M(a))$ small bodies, at the boundary of which transmission boundary conditions are imposed, is studied.

Smallness of the bodies means that $k a<<1$, where $a$ is the characteristic dimension of the body and $k=\frac{2 \pi}{\lambda}$ is the wave number in the medium in which small bodies are embedded.

Explicit asymptotic formulas are derived for the field scattered by a single small scatterer of an arbitrary shape.

Equation for the effective field is derived in the limit as $a \rightarrow 0$ while $M(a) \rightarrow \infty$ at a suitable rate.
\end{abstract}

Mathematics Subject Classification: 35J05; 74J20; 78A45;

PACS 03.04.Kf; 03.50.De; 41.20.Jb; 71.36.+c

Keywords: Transmission boundary conditions; many-body wave scattering; effective field.

\section{Introduction}

There is a large literature on "homogenization", which deals with the properties of the medium in which other materials is distributed. Quite often it is assumed that the medium is periodic, and homogenization is considered in the framework of G-convergence $([4,[5])$. In most cases, one considers elliptic or parabolic problems with elliptic operators positive-difinite and having discrete spectrum.

The author has developed a theory of wave scattering by many small particles embedded in an inhomogeneous medium ([8]-[13]). One of the pratically important consequences of his theory was a derivation of the equation for the effective (self-consistent) field in the limiting medium, obtained in the limit 
$a \rightarrow 0, M=M(a) \rightarrow \infty$, where $a$ is the characteristic size of a small particle, and $M(a)$ is the total number of the embedded particles.

The theory was developed in [8]-[13] for boundary conditions (bc) on the surfaces of small bodies, which include the Dirichlet bc, $\left.u\right|_{S_{m}}=0$, where $S_{m}$ is the surface of the $m$-th particle $D_{m}$, the impedance bc, $\left.\zeta_{m} u\right|_{S_{m}}=\left.u_{N}\right|_{S_{m}}$, where $N$ is the unit normal to $S_{m}$, pointing out of $D_{m}, \zeta_{m}$ is the boundary impedance, and the Neumann bc, $\left.u_{N}\right|_{S_{m}}=0$.

The novelty in this paper is the development of a similar theory for the transmission (interface) bc:

$$
\rho_{m} u_{N}^{+}=u_{N}^{-}, \quad u^{+}=u^{-} \quad \text { on } S_{m}, 1 \leq m \leq M .
$$

Here $0 \leq \rho_{m} \neq 1$ is a constant, $+(-)$ denotes the limit of $\frac{\partial u}{\partial N}$, from inside (outside) of $D_{m}$.

The physical meaning of the transmission boundary conditions is the continuity of the pressure and the normal component of the velocity across the boundaries of the discontinuity of the density. One may think about problem (1)-(5) as of the problem of acoustic wave scattering by many small bodies.

The essential novelty of the theory, developed in this paper, is the asymptotically exact, as $a \rightarrow 0$, treatment of the one-body and many-body scalar wave scattering problem in the case of small scatterers on the boundaries of which the transmission boundary conditions are imposed. An analytic explicit asymptotic formula for the field scattered by one small body is derived. An integral equation for the limiting effective field in the medium, in which many small bodies are embedded, is derived in the limit $a \rightarrow 0$ and $M(a) \rightarrow \infty$, where $M(a)$ is the total number of the embedded small bodies (particles), and $M=M(a)$ tends to infinity at a suitable rate as $a \rightarrow 0$.

For the problem with the number $M$ of particles not large, say, less than 5000 , our theory gives an efficient numerical method for solving many-body wave scattering problem.

For the problem with $M$ very large, say, larger than $10^{5}$, the solution to many-body wave scattering problem consists in numerical solution of the integral equation for the limiting field in the medium, in which small particles are embedded. The solution to this equation approximates the solution to the many-body wave scattering problem with high accuracy.

Our approach is quite different from the approach developed in homogenization theory, we do not assume periodicity in the location of the small scatterers. Our results are of interest also in the case when the number of scatterers is not large, so the homogenization theory is not applicable. 
Let us formulate the scattering problem we are treating.

$$
\begin{aligned}
& \text { Let } \Omega:=\bigcup_{m=1}^{M} D_{m}, \quad \Omega^{\prime}=\mathbb{R}^{3} \backslash \Omega, \\
& \left(\nabla^{2}+k^{2}\right) u=0 \quad \text { in } \Omega^{\prime}, \\
& \left(\nabla^{2}+k_{m}^{2}\right) u=0 \quad \text { in } D_{m}, \quad 1 \leq m \leq M, \\
& u=u_{0}+v, u_{0}=e^{i k \alpha \cdot x}, \quad \alpha \in S^{2}, S^{2} \text { is a unit sphere in } \mathbb{R}^{3}, \\
& r\left(\frac{\partial v}{\partial r}-i k v\right)=o(1), \quad r \rightarrow \infty .
\end{aligned}
$$

We assume that $\rho_{m}, k$ and $k_{m}^{2}$ are fixed given positive constants, and the surfaces $S_{m}$ are smooth. A sufficient smoothness condition is $S_{m} \in C^{1, \mu}, \mu \in$ $(0,1)$, where $S_{m}$ in local coordinates is given by a continuously differentiable function whose first derivatives are Hölder-continuous with exponent $\mu$.

We assume that $x_{m} \in D_{m}$ is a point inside $D_{m}, a=\frac{1}{2} \operatorname{diam} D_{m}, d=$ $O\left(a^{\frac{1}{3}}\right)$ is the distance between the neighboring particles, $\mathcal{N}(\Delta)=\sum_{x_{m} \in \Delta} 1$, is the number of particles in an arbitrary open set $\Delta$, the domains $D_{m}$ are not intersecting, and

$$
\mathcal{N}(\Delta)=\frac{1}{V} \int_{\Delta} N(x) d x[1+o(1)], \quad a \rightarrow 0,
$$

where $N(x) \geq 0$ is a function which is at our disposal, $V$ is the volume of one small body, $V=O\left(a^{3}\right)$. If $D_{m}$ are balls of radius $a$, then $V=\frac{4 \pi a^{3}}{3}$.

It is proved in [6] that problem (17)-(5) has a unique solution.

We study wave scattering by a single small body in Section 2. In other words, we study in Section 2 problem (11)-(5) with $M=1$. The basic results of this Section are formulated in Theorem 1.

In section 3 wave scattering by many small bodies is considered. The basic results of this Section are formulated in Theorem 2. We always assume that

$$
k a<<1, \quad d=O\left(a^{\frac{1}{3}}\right) .
$$

\section{Wave scattering by one small body}

Let us look for the solution to problem (1)-(5) with $M=1$ of the form

$$
u(x)=u_{0}(x)+\int_{S} g(x, t) \sigma(t) d t+\varkappa \int_{D} g(x, y) u(y) d y,
$$

where $S=S_{1}, D=D_{1}, t$ is a point on the surface $S, d t$ is the element of the surface area,

$$
\varkappa:=k_{1}^{2}-k^{2}, \quad g(x, y):=\frac{e^{i k|x-y|}}{4 \pi|x-y|},
$$


and $\sigma(t)$ is to be found so that conditions (1) are satisfied. If the pair of functions $\sigma(t)$ and $u(x)$ can be found uniquely from equations (8) and (10), see below, then the scattering problem is solved.

For any $\sigma \in C^{0, \mu_{1}}, \mu_{1} \in(0,1]$, where $C^{0, \mu_{1}}$ is the set of Hölder-continuous functions with Hölder's exponent $\mu_{1}$, the solution to equation (8) satisfies equations (2) and (3) with $M=1$, and equations (4) and (5). This is easily checked by a direct calculation. The second condition (1) is also satisfied. To satisfy the first condition in equations (1) with $\rho_{1}=\rho$, one has to satisfy the following equation

$$
(\rho-1) u_{0_{N}}+\rho \frac{A \sigma+\sigma}{2}-\frac{A \sigma-\sigma}{2}+(\rho-1) \frac{\partial}{\partial N_{s}} B u=0
$$

where

$$
A \sigma=2 \int_{S} \frac{\partial g(s, t)}{\partial N_{S}} \sigma(t) d t, \quad B u=\varkappa \int_{D} g(x, y) u(y) d y
$$

and the well-known formulas for the limiting values of the normal derivatives of the single-layer potential $T \sigma:=\int_{S} g(x, t) \sigma(t) d t$ on $S$ from inside and outside $D$ was used.

In [6] one finds a proof of the following existence and uniqueness result. Let $H^{2}(D)$ denote the usual Sobolev space of functions twice differentiable in $L^{2}$-sense.

Proposition 1. The system of equations (8) and (10) for the unknown functions $\sigma$ on $S$ and $u(x)$ in $D$ has a solution and this solution is unique in $C^{0, \mu_{1}} \times H^{2}(D)$. $\mathbb{R}^{3}$.

If the solution $\left\{\sigma,\left.u(x)\right|_{x \in D}\right\}$ is found, then formula (8) defines $u=u(x)$ in

Let us rewrite 10 as

$$
\sigma=\lambda A \sigma+2 \lambda B_{1} u+2 \lambda u_{0_{N}}
$$

where

$$
\lambda=\frac{1-\rho}{1+\rho}, \quad B_{1} u=\varkappa \frac{\partial}{\partial N_{s}} \int_{D} g(x, y) u(y) d y .
$$

If $\rho \in(0, \infty)$ then $\lambda \in(-1,1)$. Let us now use the first assumption (7), that 
is, the smallness of $a$. One has:

$$
\begin{aligned}
& g(s, t)=g_{0}(s, t)(1+O(k a)), \quad a \rightarrow 0 ; \quad g_{0}(s, t)=\frac{1}{4 \pi|s-t|}, \\
& \frac{\partial}{\partial N_{s}} \frac{e^{i k|s-t|}}{4 \pi|s-t|}=\frac{\partial g_{0}}{\partial N_{s}}\left(1+O\left((k a)^{2}\right)\right), \quad a \rightarrow 0, \\
& \text { so } A=A_{0}\left(1+O\left((k a)^{2}\right)\right), \quad a \rightarrow 0 ; A_{0}:=\left.A\right|_{k=0}, \\
& B=B_{0}(1+O(k a)), \quad B_{0} u=\varkappa \int_{D} g_{0}(x, y) u(y) d y, \\
& B_{1} u=\varkappa \int_{D} \frac{\partial g_{0}(s, y)}{\partial N} u(y) d y\left(1+O\left(k^{2} a^{2}\right)\right):=\varkappa B_{10} u\left(1+O\left(k^{2} a^{2}\right)\right) .
\end{aligned}
$$

It follows from equation (8) that

$$
u(x)=u_{0}(x)+\frac{e^{i k\left|x-x_{1}\right|}}{\left|x-x_{1}\right|}\left(\frac{1}{4 \pi} \int_{S} e^{-i k \beta \cdot t} \sigma(t) d t+\frac{\varkappa}{4 \pi} u_{1} V_{1}\right), \quad\left|x-x_{1}\right|>>a,
$$

where $V_{1}$ is the volume of $D=D_{1}, V_{1}=\operatorname{vol}\left(D_{1}\right):=\left|D_{1}\right|, u_{1}:=u\left(x_{1}\right)$, $\beta:=\frac{x-x_{1}}{\left|x-x_{1}\right|}$. The point $x_{1} \in D$ can be chosen as we wish. For one scatterer it is convenient to choose the origin at the point $x_{1}$ so that $x_{1}=0$.

We did not keep the factor $e^{-i k \beta \cdot x}$ in the integral over $D$ because $e^{-i k \beta \cdot x}=$ $1+O(k a)$, and

$$
\int_{D} e^{-i k \beta \cdot y} u(y) d y=u_{1} V_{1}(1+O(k a)), \quad a \rightarrow 0 .
$$

However, it will be proved that this factor under the surface integral can not be dropped because

$$
\int_{S} e^{-i k \beta \cdot t} \sigma(t) d t=\int_{S} \sigma(t) d t-i k \beta_{p} \int_{S} t_{p} \sigma(t) d t+O\left(a^{4}\right),
$$

where over the repeated indices here and throughout this paper summation is understood, and the second integral in the right-hand side of (21) is $O\left(a^{3}\right)$, as $a \rightarrow 0$, that is, it is of the same order of smallness as the the first integral $Q:=\int_{S} \sigma(t) d t$. The last statement will be proved later.

With the notations

$$
Q:=\int_{S} \sigma(t) d t, \quad Q_{1}:=\int_{S} e^{-i k \beta \cdot t} \sigma(t) d t,
$$

the expression

$$
A(\beta, \alpha):=\frac{Q_{1}}{4 \pi}+\frac{\varkappa}{4 \pi} u_{1} V_{1}, \quad V_{1}:=V:=|D|, \quad u_{1}:=u\left(x_{1}\right),
$$


is the scattering amplitude, $\alpha$ is the unit vector in the direction of the incident wave $u_{0}=e^{i k \alpha \cdot x}, \beta$ is the unit vector in the direction of the scattered wave. Let us prove that

$$
-i k \beta_{p} \int_{S} t_{p} \sigma(t) d t=O\left(a^{3}\right)
$$

and therefore, the second integral in the right-hand side of equation 21 cannot be dropped.

It follows from equation (8) that

$$
u(x) \sim u_{0}(x)+g\left(x, x_{1}\right) Q_{1}+\varkappa g\left(x, x_{1}\right) u\left(x_{1}\right) V_{1}, \quad\left|x-x_{1}\right| \geq d \gg a,
$$

where $\sim$ means asymptotic equivalence as $a \rightarrow 0$.

Formula (25) can be used for calculating $u(x)$ if two quantities $Q_{1}$ and $u_{1}:=$ $u\left(x_{1}\right)$ are found.

Let us derive asymptotic formulas for these quantities as $a \rightarrow 0$. Integrate equation 12 over $S$ and get

$$
Q=2 \lambda \int_{S} u_{0_{N}} d s+\lambda \int_{S} A \sigma d t+2 \lambda \int_{S} B_{1} u d s
$$

Use formulas (14)-(18), the following formula (see [7], p.96):

$$
\int_{S} A_{0} \sigma d s=-\int_{S} \sigma d s
$$

and the Divergence theorem, to rewrite equation $(26)$ as

$$
Q=2 \lambda \int_{D} \nabla^{2} u_{0} d x-\lambda Q+2 \lambda \varkappa \int_{D} d x \nabla_{x}^{2} \int_{D} g(x, y) u(y) d y
$$

Since

$$
\nabla^{2} u_{0}=-k^{2} u_{0} ; \quad \nabla_{x}^{2} g(x, y)=-k^{2} g(x, y)-\delta(x-y),
$$

equation (28) takes the form

$$
(1+\lambda) Q=2 \lambda \nabla^{2} u_{0}\left(x_{1}\right) V_{1}-2 \lambda k^{2} \varkappa \int_{D} d x \int_{D} g(x, y) u d y-2 \lambda \varkappa \int_{D} u(x) d x
$$

Let us use the following estimates:

$$
\begin{aligned}
& \int_{D} u(x) d x=u_{1} V_{1}(1+o(1)), \quad a \rightarrow 0 ; \quad u_{1}:=u\left(x_{1}\right) \\
& \int_{D} d x \int_{D} g(x, y) u(y) d y=\int_{D} d y u(y) \int_{D} d x g(x, y)=O\left(a^{5}\right) \\
& \int_{D} g(x, y) d x=O\left(a^{2}\right), \quad \forall y \in D .
\end{aligned}
$$


From equations $30-33$ it follows that

$$
Q \sim \frac{2 \lambda}{1+\lambda} V_{1} \nabla^{2} u_{01}-\frac{2 \lambda \varkappa}{1+\lambda} V_{1} u_{1}, \quad a \rightarrow 0,
$$

where

$$
\nabla^{2} u_{01}=\left.\nabla^{2} u_{0}(x)\right|_{x=x_{1}} .
$$

Let us now integrate equation (8) over $D$ and use estimate (31) to obtain

$$
u_{1} V_{1}=u_{01} V_{1}+\int_{S} d t \sigma(t) \int_{D} g(x, t) d x+\varkappa \int_{D} d y u(y) \int_{D} g(x, y) d x
$$

If $D$ is a ball of radius $a$, then one can easily check that

$$
\int_{D} g(x, t) d x \sim \int_{D} g_{0}(x, t) d x=\frac{a^{2}}{3}, \quad|t|=a, \quad a \rightarrow 0 .
$$

In general, one has

$$
\int_{D} g(x, y) d x=O\left(a^{2}\right), \quad y \in D, \quad a \rightarrow 0 .
$$

If $D$ is a ball of radius $a$, then equations (36)-(38) imply

$$
u_{1}=u_{01}+Q \frac{a^{2}}{3 \frac{4 \pi a^{3}}{3}}+\varkappa u_{1} O\left(a^{2}\right), \quad a \rightarrow 0 .
$$

Consequently,

$$
u_{1} \sim u_{01}+O\left(a^{2}\right), \quad a \rightarrow 0,
$$

because $Q=O\left(a^{3}\right)$.

Indeed, from equations 34 and 40 one gets

$$
Q \sim V_{1}(1-\rho)\left[\nabla^{2} u_{01}-\varkappa u_{01}\right]
$$

where we took into account that

$$
\frac{2 \lambda}{1+\lambda}=1-\rho
$$

the relation $u_{1} \sim u_{01}$ as $a \rightarrow 0$, see equation (40), and neglected the terms of higher order of smallness. It follows from equation (41) that

$$
Q=O\left(a^{3}\right)
$$

From equations 40 and 41 one obtains

$$
u_{1} \sim u_{01}, \quad a \rightarrow 0 .
$$


Let us now estimate $Q_{1}$. One has

$$
Q_{1}=\int_{S} \sigma(t) d t-i k \beta_{p} \int_{S} t_{p} \sigma(t) d t
$$

up to the terms of the higher order of smallness as $a \rightarrow 0$, and summation is understood over the repeated indices. It turns out that the integral

$$
I:=\int_{S} t_{p} \sigma(t) d t
$$

is of the same order, namely $O\left(a^{3}\right)$, as $Q=\int_{S} \sigma(t) d t$.

Let us check that the integral

$$
J:=\int_{S} d t t_{p} \frac{\partial}{\partial N} \int_{D} g(t, y) u(y) d y=O\left(a^{4}\right)
$$

as $a \rightarrow 0$, and, therefore, can be neglected compared with $I$. Indeed, $u=O(1)$, $\int_{D} \frac{\partial}{\partial N} g(t, y) d y=O(a)$, and $\int_{S} t_{p} d t=O\left(a^{3}\right)$. Thus, $J=O\left(a^{4}\right)$.

Define the function $\sigma_{q}, q=1,2,3$, as the unique solution to the equation

$$
\sigma_{q}=\lambda A \sigma_{q}-2 \lambda N_{q}
$$

Since $\lambda=(1-\rho) /(1+\rho)$, and $\rho>0$, one concludes that $\lambda \in(-1,1)$, and it is known (see, for example, [7]) that the operator $A$ is compact in $L^{2}(S)$ and does not have characteristic values in the interval $(-1,1)$. This and the Fredholm alternative imply that equation (47) has a solution and this solution is unique.

Let us prove that $\int_{S} \sigma_{q}(t) d t=O\left(a^{3}\right)$. To do this, integrate equation 47) over $S$, take into account formula (27), the relation $\left(A-A_{0}\right) \sigma_{q}=O\left(a^{3}\right)$, and obtain

$$
(1+\lambda) \int_{S} \sigma_{q}(t) d t=-2 \lambda \int_{S} N_{q} d t+O\left(a^{3}\right)=O\left(a^{3}\right),
$$

because $\int_{S} N_{q} d t=0$ by the Divergence theorem.

Define the tensor

$$
\beta_{p q}:=\beta_{p q}(\lambda):=V_{1}^{-1} \int_{S} t_{p} \sigma_{q}(t) d t, \quad p, q=1,2,3 .
$$

This tensor is similar to the tensor $\beta_{p q}$ defined in [7], p. 62, by a similar formula with $\lambda=1$. In this case $\beta_{p q}$ is the magnetic polarizability tensor of a superconductor $D$ placed in a homogeneous magnetic field directed along the unit Cartesian coordinate vector $e_{q}$ (see [7], p. 62). In [7] analytic formulas are given for calculating $\beta_{p q}$ with an arbitrary accuracy. 
One may neglect the term $B_{1} u$ in equation 12 (because this term is $O\left(a^{4}\right)$ ), take into account definition (48), and get

$$
\int_{S} t_{p} \sigma(t) d t=-\beta_{p q} \frac{\partial u_{0}}{\partial x_{q}} V,
$$

where $V:=V_{1}$, and summation is understood over $q$.

Consequently, one can rewrite (45) as

$$
Q_{1}=(1-\rho) V_{1}\left[\nabla^{2}\left(u_{0}\left(x_{1}\right)-\varkappa u_{0}\left(x_{1}\right)\right]+i k \beta_{p q} \frac{\partial u_{0}}{\partial x_{q}} \beta_{p} V_{1}, \quad \beta:=\frac{x-x_{1}}{\left|x-x_{1}\right|},\right.
$$

and $(x)_{p}:=x \cdot e_{p}$ is the $p$-th Cartesian coordinate of the vector $x$.

Formula (19) can be written as

$u(x)=u_{0}(x)+g\left(x, x_{1}\right)\left((1-\rho)\left[\nabla^{2} u_{0}\left(x_{1}\right)-\varkappa u_{0}\left(x_{1}\right)\right]+i k \beta_{p q} \frac{\partial u_{0}\left(x_{1}\right)}{\partial x_{1, q}} \beta_{p}+\varkappa u_{0}\left(x_{1}\right)\right) V_{1}$.

Here one sums over the repeated indices, $\left|x-x_{1}\right|>>a$, and $\frac{\partial u_{0}\left(x_{1}\right)}{\partial x_{1, q}}:=$ $\left.\frac{\partial u_{0}(y)}{\partial y_{q}}\right|_{y=x_{1}}, q=1,2,3, y=\left(y_{1}, y_{2}, y_{3}\right)$.

Formulas (41), (43), (44) are valid for small $D$ of an arbitrary shape. Let us formulate the results of this Section in Theorem 1.

Theorem 1. Assume that $k a \ll 1, k_{1}, k$, and $\rho$ are positive constants. Then the scattering problem (1)-(5) has a unique solution. This solution has the form (8) and can be calculated by formula (51) in the region $\left|x-x_{1}\right|>>a$ up to the terms of order $O\left(a^{4}\right)$ as $a \rightarrow 0$, where $a=0.5$ diam $D, \varkappa=k_{1}^{2}-k^{2}$, $V_{1}=\operatorname{vol} D, \beta=\frac{x-x_{1}}{\left|x-x_{1}\right|}, \beta_{p q}$ is defined in equation (48), and $O\left(a^{4}\right)$ does not depend on $x$.

\section{$3 \quad$ Wave scattering by many small bodies}

Assume that the distribution of small bodies is given by equation (6), and that there are $M=M(a)$ non-intersecting small bodies $D_{m}$ of size $a$. For simplicity we assume that $D_{m}$ is a ball of radius a, centered at $x_{m}$. There is an essential novel feature in the theory, developed in this paper compared with the one developed in [8], [9], [12], namely, the scattered field was much larger, as $a \rightarrow 0$ in the above papers. For example, for the impedance boundary condition, $u_{N}=\zeta u$ on $S$, the scattered field is $O\left(a^{2}\right)$, and for the Dirichlet boundary condition, $u=0$ on $S$, the scattered field is $O(a)$.

For the Neumann boundary condition the scattered field is $O\left(a^{3}\right)$. We have the same order of smallness of the scattered field, $O\left(a^{3}\right)$, for the problem with the transmission boundary condition because $V_{1}=O\left(a^{3}\right)$. The basic role in Section 3 is played by formula (51). We assume that the distance $d$ between 
neighboring bodies (particles) is much larger than $a, d>>a$, but there can be many small particles on the wavelength, and the interaction of the scattered waves (multiple scattering) is essential and cannot be neglected.

This assumption effectively means that the function $N(x)$ in $(6)$ has to be small, $N(x)<<1$. Indeed, if on a segment of unit length there are small particles placed at a distance $d$ between neighboring particles, then there are $O\left(\frac{1}{d}\right)$ particles on this unit segment, and $O\left(\frac{1}{d^{3}}\right)$ in a unit cube $C_{1}$. Since $V=O\left(a^{3}\right)$, by formula (6) one gets

$$
\frac{1}{O\left(a^{3}\right)} \int_{C_{1}} N(x) d x=O\left(\frac{1}{d^{3}}\right) \text {. }
$$

Therefore $d>>a$ can hold only if $\left(\int_{C_{1}} N(x) d x\right)^{\frac{1}{3}}=O\left(\frac{a}{d}\right)<<1$.

Let us look for the (unique) solution to problem (1)-(5) with $1 \leq m \leq M=$ $M(a)$ of the form

$$
u(x)=u_{0}(x)+\sum_{m=1}^{M} \int_{S_{m}} g(x, t) \sigma_{m}(t) d t+\sum_{m=1}^{M} \varkappa_{m} \int_{D_{m}} g(x, y) u(y) d y .
$$

Keeping the main terms in this equation, as $a \rightarrow 0$, one gets

$$
\begin{aligned}
u(x) & =u_{0}(x)+\sum_{m=1}^{M} g\left(x, x_{m}\right)\left(Q_{m}-i k \frac{\left(x-x_{m}\right)_{p}}{\left|x-x_{m}\right|} \int_{S_{m}} t_{p} \sigma_{m}(t) d t\right)+ \\
& +\sum_{m=1}^{M} \varkappa_{m} g\left(x, x_{m}\right) u_{e}\left(x_{m}\right) V_{m}, \quad Q_{m}:=\int_{S_{m}} \sigma_{m}(t) d t, \quad a \rightarrow 0
\end{aligned}
$$

where we have used formula (51) for the scattered field by every small particle, replaced $u_{0}$ by the effective field $u_{e}$, acting on every particle, and took into account that $\beta:=\beta_{m}:=\frac{x-x_{m}}{\left|x-x_{m}\right|}$. By $\left(x-x_{m}\right)_{p}$ the $p$-th component of vector $\left(x-x_{m}\right)$ is denoted.

The effective (self-consisted) field $u_{e}$, acting on $j$-th particle, is defined as:

$$
\begin{aligned}
& u_{e}(x)=u_{0}(x)+\sum_{m=1, m \neq j}^{M} g\left(x, x_{m}\right)\left(\left(1-\rho_{m}\right)\left[\nabla^{2} u_{e}\left(x_{m}\right)-\varkappa_{m} u_{e}\left(x_{m}\right)\right]+\right. \\
& \left.i k \beta_{p q}^{(m)} \frac{\partial u_{e}}{\partial x_{q}} \frac{\left(x-x_{m}\right)_{p}}{\left|x-x_{m}\right|}\right) V_{m}+\sum_{m=1, m \neq j}^{M} \varkappa_{m} g\left(x, x_{m}\right) u_{e}\left(x_{m}\right) V_{m}, \quad\left|x-x_{j}\right| \sim a .
\end{aligned}
$$

Setting $x=x_{j}$ in equation (54), one gets a linear algebraic system for the unknowns $u_{j}:=u_{e}\left(x_{j}\right), 1 \leq j \leq M, \nabla^{2} u_{j}:=\nabla^{2} u_{e}\left(x_{j}\right)$, and $\frac{\partial u_{e}\left(x_{j}\right)}{\partial x_{j, p}}$. Here 
$x_{j, p}$ is the $p$-th component of the vector $x_{j}, p=1,2,3$. Differentiating (54) with respect to $x_{j, p}, p=1,2,3$, and then setting $x=x_{j}$, one obtains a linear algebraic system for the $5 M$ unknowns $u_{j}, \nabla^{2} u_{j}$, and $\frac{\partial u_{e}\left(x_{j}\right)}{\partial x_{j, p}}, 1 \leq j \leq M$, $1 \leq p \leq 3$

This linear algebraic system one gets if one solves by a collocation method the following integral equation

$$
\begin{aligned}
& u(x)=u_{0}(x)+\int_{D} g(x, y)\left[(1-\rho)\left(\nabla^{2}-K^{2}(y)+k^{2}\right) u(y)+\right. \\
& \left.i k \beta_{p q}(y, \lambda) \frac{\partial u(y)}{\partial y_{q}} \frac{(x-y)_{p}}{|x-y|}+\left(K^{2}(y)-k^{2}\right) u(y)\right] N(y) d y .
\end{aligned}
$$

In the above equation the function $\beta_{p q}(y, \lambda)$ is defined as

$$
\beta_{p q}(y, \lambda)=\lim _{a \rightarrow 0} \frac{\sum_{x_{m} \in \Delta_{p}} \beta_{p q}^{(m)}}{\mathcal{N}\left(\Delta_{p}\right)}
$$

where $y=y_{p} \in \Delta_{p}$, and tensor $\beta_{p q}^{(m)}=\beta_{p q}^{(m)}(\lambda)$ is defined in (48). Convergence of the collocation method was proved in [12].

Equation (55) is a non-local integrodifferential equation for the limiting effective field in the medium in which many small bodies are embedded.

This is a novel result. The original scattering problem (1)-(5) has been formulated in terms of local differential operators.

In the derivation of equation (55) from equation (54) we have assumed that $\rho_{m}=\rho$ does not depend on $m$, took into account that $\varkappa_{m}^{2}$ becomes in the limit $K^{2}(y)-k^{2}$, and denoted by $K^{2}(y)$ a continuous function in $D$ such that $K^{2}\left(x_{m}\right)=k_{m}^{2}$. As $a \rightarrow 0$ the function $K^{2}(y)$ is uniquely defined because the set $\left\{x_{m}\right\}_{m=1}^{M(a)}$ becomes dense in $D$ as $a \rightarrow 0$.

To derive equation (55) from equation (54) we argue as follows. Consider a partition of $D$ into a union centered at the points $y_{p}$ of $P$ non-intersecting cubes $\Delta_{p}$, of size $b(a), b(a)>>d$, so that each cube contains many small bodies, $\lim _{a \rightarrow 0} b(a)=0$. Let us demonstrate the passage to the limit $a \rightarrow 0$ in the sums in equation (54) using the first sum as an example. Write the first sum in (54) as

$$
\begin{aligned}
& \sum_{m \neq j} g\left(x, x_{m}\right)\left(1-\rho_{m}\right)\left[\nabla^{2} u_{e}\left(x_{m}\right)-\kappa_{m} u_{e}\left(x_{m}\right)\right] V_{m} \\
& =\sum_{p=1}^{P} g\left(x, y_{p}\right)\left(1-\rho_{p}\right)\left[\nabla^{2} u_{e}\left(y_{p}\right)-\kappa_{p} u_{e}\left(y_{p}\right)\right] V_{m} \sum_{x_{m} \in \Delta_{p}} 1 \\
& =\sum_{p=1}^{P} g\left(x, y_{p}\right)\left(1-\rho_{p}\right)\left[\nabla^{2} u_{e}\left(y_{p}\right)-\kappa_{p} u_{e}\left(y_{p}\right)\right] N\left(y_{p}\right)\left|\Delta_{p}\right|(1+o(1)),
\end{aligned}
$$


where we have used formula (6), took into account that diam $\Delta_{p} \rightarrow 0$ as $a \rightarrow 0$, wrote formula (6) as

$$
V \sum_{x_{m} \in \Delta_{p}} 1=V \mathcal{N}\left(\Delta_{p}\right)=N\left(y_{p}\right)\left|\Delta_{p}\right|(1+o(1)), \quad a \rightarrow 0
$$

and used the Riemann integrability of the functions involved, which holds, for example, if these functions are continuous. By $\rho_{p}$ we denote the value $\rho\left(y_{p}\right)$, where $\rho(y)$ is a continuous function.

The sum in (56) is the Riemannian sum for the integral

$$
\int_{D} g(x, y)(1-\rho(y))\left[\nabla^{2} u(y)-K^{2}(y) u(y)+k^{2} u(y)\right] N(y) d y .
$$

Similarly one treats the other sums in (54) and obtains in the limit $a \rightarrow 0$ equation (55).

Let us formulate the results of this Section.

Theorem 2. Assume that conditions (6) and (7) hold. Then, as $a \rightarrow 0$, the effective field, defined by equation (54), has a limit $u(x)$. The function $u(x)$ solves equation (55).

\section{Conclusions}

In this paper wave scattering by one and many small bodies of an arbitrary shape is studied in the case when the boundary conditions are transmission ones. Explicit analytic formulas are derived for the field scattered by a single body, see Theorem 1.

Equation for the effective field in the medium where many small bodies are distributed is derived. The result is formulated in Theorem 2.

One concludes that the wave scattered by a small particle subject to transmission boundary condition can be calculated by formula (51). If there are many small particles distributed in a bounded domain according to formula (6), then one conludes that the limiting effective field in such a medium satisfies equation (55) and can be calculated by solving a linear algebraic system which one obtains from formula (54) by setting $x=x_{j}$ in equation (54). 


\section{References}

[1] M.Andriychuk, A.G.Ramm: Numerical solution of many-body wave scattering problem for small particles and creating materials with desired refraction coefficient, Chapter in the book "Numerical simulation of physical and engineering processes, InTech, Vienna, 2011, pp. 1-28. (editor J.Awrejcewicz), ISBN 978-953-307-620-1.

[2] C. Bohren, D. Huffman: Absorption and scattering of light by small particles, Wiley, New York, 1983.

[3] H. van de Hulst: Light scattering by small particles, Dover, New York, 1981.

[4] V.Jikov, S.Kozlov, O.Oleinik: Homogenization of differential and integral functionals, Springer, Berlin, 1994.

[5] V.Marchenko, E.Khruslov: Homogenization of partial differential equations, Birkhäuser, Basel, 2006.

[6] A.G. Ramm: Scattering by obstacles, D.Reidel, Dordrecht, 1986.

[7] A.G. Ramm: Wave scattering by small bodies of arbitrary shapes, World Scientific, New Jersey, 2005.

[8] A.G. Ramm: Many-body wave scattering by small bodies and applications, J. Math. Phys., 48, 103511 (2007).

[9] A.G. Ramm: Wave scattering by many small particles embedded in a medium, Phys. Lett. A, 372/17, 3064 (2008),

[10] A.G. Ramm: A method for creating materials with a desired refraction coefficient, Internat, Journ. Mod. Phys B, 24, 5261 (2010).

[11] A.G. Ramm: Materials with a desired refraction coefficient can be created by embedding small particles into a given material, International Journal of Structural Changes in Solids (IJSCS), 2, 17 (2010).

[12] A.G. Ramm: Wave scattering by many small bodies and creating materials with a desired refraction coefficient, Afrika Matematika, 22, 33 (2011).

[13] A.G. Ramm: Scattering of scalar waves by many small particles, AIP Advances, 1, 022135, (2011). 